Recherches en didactique des langues et des cultures

Les cahiers de l'Acedle

$15-2 \mid 2018$

Quelles médiations en didactique des langues et des cultures?

\title{
Enseigner les langues-cultures dans une optique de genre
}

Une étude des stéréotypes dans les manuels de français et d'italien langue seconde ou étrangère

Edith Cognigni et Silvia Vecchi

\section{OpenEdition}

Journals

Édition électronique

URL : http://journals.openedition.org/rdlc/4103

DOI : $10.4000 /$ rdlc. 4103

ISSN : 1958-5772

Éditeur

ACEDLE

Référence électronique

Edith Cognigni et Silvia Vecchi, « Enseigner les langues-cultures dans une optique de genre »,

Recherches en didactique des langues et des cultures [En ligne], 15-2 | 2018, mis en ligne le 02 juin 2018, consulté le 30 avril 2019. URL : http://journals.openedition.org/rdlc/4103; DOI : 10.4000/rdlc.4103

Ce document a été généré automatiquement le 30 avril 2019.

\section{$\Theta \Theta \Theta \Theta$}

Recherches en didactique des langues et des cultures is licensed under a Creative Commons AttributionNonCommercial-NoDerivatives 4.0 International License 


\section{Enseigner les langues-cultures dans une optique de genre}

Une étude des stéréotypes dans les manuels de français et d'italien langue seconde ou étrangère

\section{Edith Cognigni et Silvia Vecchi}

1 Des recherches ${ }^{1}$ conduites au cœur des systèmes éducatifs de nombreux pays européens soulignent la présence des disparités de réussite, d'attitudes ou de motivation entre les filles et les garçons, en particulier dans le domaine des langues où l'on enregistre un écart numérique considérable en faveur du genre féminin aussi bien du côté des élèves que du corps enseignant (Commission Européenne, 2010). Plus le niveau de formation s'élève, plus la fréquentation de la classe de langue semble évoluer vers un contexte social au féminin qui peut aussi avoir un impact incisif sur la didactique elle-même, au point de déterminer une féminisation de l'enseignement des langues (Schmenk, 2007).

Il est donc évident que la prise de conscience de la part des enseignant.e.s de la dimension de genre impliquée par la langue-culture enseignée et surtout de sa réception chez les apprenant.e.s s'avère un facteur important pour promouvoir un climat d'équité entre les sexes. La notion d'équité est ici adoptée pour souligner la nécessité de repenser l'enseignement des langues-cultures dans une optique de genre qui ne neutralise pas les différences entre filles et garçons mais qui, au contraire, les prenne en charge comme catalyseurs d'une réflexion sur la diversité au sens large, qu'il s'agisse d'une différence sexuelle, socioculturelle ou individuelle. Comme le soulignent Magno et Silova (2007: 649) :

The term equality is used to refer to a situation in which students receive the same quality of teaching and enjoy the same type of school facilities, textbooks, and teaching materials. [...] Gender equity, in contrast, is based on the principle of social justice and refers to the guarantee of fair educational outcomes, regardless of sex differences ${ }^{2}$.

3 Par conséquent, il est important de mettre en œuvre des approches qui mobilisent les besoins et les talents aussi bien des filles que des garçons, au-delà d'une vision stéréotypée et préconstituée des rôles masculin et féminin, dans la société 
d'appartenance comme dans celle dont on étudie la langue. L'enseignement/ apprentissage des langues étrangères, grâce à l'effet de distanciation par rapport à la langue-culture maternelle, est envisagé comme un lieu privilégié pour la comparaison, le méta-discours, le « retour » sur la langue-culture maternelle elle-même qui est en général vécue comme une évidence naturelle alors qu'elle est une construction sociale et culturelle.

Nos réflexions sont inspirées des études de genre appliquées à l'enseignement des langues-cultures produites en contexte européen et nord-américain (en particulier Baurens 2006, 2009; Decke-Cornill \& Volkmann, 2007 ; Pavlenko, 2004) et se fondent en particulier sur ce que Pavlenko définit comme "pédagogies féministes critiques » (ibid: 53), un domaine d'études à l'intersection du féminisme poststructuraliste et de l'enquête critique en linguistique appliquée selon lequel le genre est un système de relations de pouvoir et de pratiques discursives, dynamiques et socialement construites, indissociables d'autres aspects constituants de l'identité sociale du sujet.

Dans la lignée de ce cadre théorique, on évoquera d'abord certaines bonnes pratiques réalisées dans le contexte des projets italiens et européens, en vue de la sensibilisation à une perspective de genre dans le champ de l'enseignement des langues; on présentera ensuite les résultats principaux d'une enquête qualitative portant sur l'analyse d'un corpus des manuels didactiques destinés à l'enseignement du français et de l'italien comme langues étrangères ou secondes ${ }^{3}$ à des publics variés dans le contexte éducatif italien.

6 Par l'approche analytique proposé, on cherchera à attirer l'attention sur la façon dont l'usage des images, des thèmes traités et de la langue participe à la production d'une représentation stéréotypée du genre. On souhaite faire émerger par cette analyse les retombées possibles de l'adoption d'une approche peu attentive ou neutre par rapport aux stéréotypes de genre, afin de sensibiliser les enseignant.e.s à une perspective critique en didactique des langues-cultures. Par "stéréotype de genre» on entend ici non seulement ce qui exclut ou minore la représentation de la composante féminine, mais toute forme de jugement ou représentation schématique qui aplatit les différences à l'intérieur des groupes, figeant ainsi les rôles et rendant indistincts les désirs et les vocations, les façons d'être et de se penser des individus ${ }^{4}$.

\section{Genre et langage dans le champ éducatif italien}

7 Cette recherche se fonde sur une série d'études et de projets menés en contexte italien qui avaient pour but de sensibiliser le système éducatif à la prise en considération d'une optique de genre.

8 Il faut rappeler en particulier le projet européen POLITE (Pari Opportunità nei Libri di Testo ), impliquant l'Italie, l'Espagne et le Portugal. Il s'agit d'un projet d'autorégulation des éditions scolaires qui est né avec le but de promouvoir une réflexion culturelle, didactique et éditoriale afin que les protagonistes de la culture et de la société soient présent.e.s dans les manuels scolaires sans discrimination de sexe. À partir du projet POLITE, un Code d'Autoréglementation a été rédigé à l'intention des éditeurs de telle sorte que la perspective de genre devienne un principe directeur pour la rédaction de futurs manuels scolaires. Le Code est associé à un Document d'Accompagnement (v. Serravalle Porzio, 2000) dans lequel sont listées les actions à mettre en œuvre afin que le manuel fasse preuve de sensibilisation aux questions de genre ${ }^{6}$. Comme le montrent des 
recherches plus récentes dans le domaine de la formation primaire en particulier (v. Biemmi, 2010), les bonnes pratiques promues par le projet POLITE sont cependant encore loin d'être généralisées.

9 Malgré ce projet constructif et sa résonance, la question de l'éducation de genre est encore un point critique - et parfois problématique (Nicola, 2016) - porté par des actions isolées d'enseignement et/ou de formation des enseignant.e.s ${ }^{7}$. Même si la recherche sur le genre en contexte éducatif est bien avancée, le lien entre éducation de genre et didactique des LS/LE est encore peu exploré ou réalisé de manière fragmentée.

On ajoute également que, bien qu'en France la réflexion inhérente à l'impact des manuels scolaires sur la représentation du genre et les rapports entre les sexes soit affirmée et que les actions d'intervention soient nombreuses ${ }^{8}$, il n'est toujours pas possible d'identifier la même conscience et attention à la différence de genre dans les manuels de FLE et d'italien LS/LE, tout au moins dans ceux qui sont diffusés sur le marché italien (cf. infra, $\$ 3$ et 4). La catégorie du genre semble faire partie d'un discours périphérique si non marginal à l'enseignement/apprentissage alors que le concept d' «intergenre » (Baurens, 2006) en gagnerait en renforcement épistémologique central en didactique des langues et dans la formation des enseignant.e.s: «il constituerait un triple apprentissage, celui de la conscience de genre (connaissance de l'effet-genre dans la classe, reconnaissance de différences de comportements, de performances entre filles et garçons); celui de la conscience de la différenciation genrée (orchestration enseignante, facilitation voire fabrication des inégalités) ; celui enfin de la conscience éthique (intervention égalitaire) » (ibid. : 252).

11 Dans cette étude, on a donc décidé de se focaliser sur les manuels de LS/LE en adoptant une démarche qui permet de confronter leurs modalités discursives sur les valeurs de genre, dans l'ordre de deux langues concernées, étant donné que ces manuels représentent des témoins exceptionnels des valeurs et des préjugés qu'une société véhicule. C'est surtout en vertu de leur influence dans la construction des discours pédagogico-didactiques et, par leur intermédiaire, de l'ordre social que les manuels jouent un rôle assez délicat pour les néo-enseignant.e.s dans la reproduction des stéréotypes de genre vis-à-vis de la culture cible .

12 On souhaite donc ouvrir quelques perspectives pour la formation des enseignant.e.s pour qu'ils/elles puissent se faire agents d'une éducation linguistique attentive à la différence de genre. On va donc se demander :

13 1) Quels stéréotypes et formes d'iniquité de genre se dégagent des manuels de français et d'italien LS/LE analysés? À travers quelles modalités verbales et non verbales sont-ils élaborés?

14 2) Quels éléments conceptuels et méthodologiques peuvent répondre à la nécessité de sensibiliser les enseignant.e.s à une «approche intergenre» à l'enseignement des langues-cultures?

\section{Méthodologie de recherche. Des modèles préconstitués à l'épreuve de l'analyse}

15 Ce travail s'appuie sur le concept-clé de "représentation sociale » dont on connaît la valeur analytique et constructive en Didactique des langues-cultures lorsque l'analyse part du terrain de la pratique didactique (Galisson, 1994). Des questions qui interpellent la 
spécificité des représentations sociales en tant que principes organisateurs de comportements, d'attitudes se caractérisant, dans une optique sociodynamique, par les rapports symboliques entre les acteurs sociaux et les variations individuelles qui interviennent dans ces rapports9.

Il s'agit de découvrir ce qui se passe dans les situations de dialogue ou à travers les illustrations : les idées et leur catégorisation qui forment un premier ordre de description vis-à-vis surtout de l'organisation sociale des relations entre les sexes. L'option méthodologique choisie cherche à faire émerger le caractère systémique des valeurs associées aux pratiques langagières des femmes et des hommes représentés dans les manuels soit de français, soit d'italien LE/LS.

Le corpus d'analyse se compose globalement de dix manuels de base de français et d'italien LE/LS, tous cherchant à répondre aux objectifs des niveaux de compétence linguistico-communicative A1, A2 et B1 du CECR (Conseil de l'Europe, 2001); ils sont réalisés par des auteur.e.s italiens et français. On a choisi des textes publiés au cours des dernières années (2003-2013) ${ }^{10}$, s'appuyant sur des conceptions didactiques récentes et largement utilisées en Italie dans les contextes de l'éducation ou de la formation linguistique. Les manuels sélectionnés sont destinés à des publics divers (adolescents et jeunes adultes, mais aussi migrants et professionnels) avec l'objectif de faire émerger des convergences éventuelles dans la représentation de genre face à des schémas macrodiscursifs diversifiés, présents dans les textes analysés.

L'analyse est de type qualitatif ${ }^{11}$ selon une approche mixte employant différents niveaux d'analyse afin de faire émerger l'ethos qui préside au choix des images, de textes oraux et écrits. Trois niveaux d'analyse se croisent : un niveau d'analyse de contenu thématique, un niveau d'analyse de discours et un niveau d'analyse en sémiotique figurative ${ }^{12}$.

S'inspirant des méthodes d'analyse thématique (Blanchet \& Gotmam, 1992), des thèmes plus sensibles à la stéréotypie de rôles sont sélectionnés : la Famille, les Professions, les Achats, les Intérêts et Loisirs. Sur la base de ces unités thématiques, on a donc examiné les textes oraux et écrits, le lexique, les images, pour explorer si ceux-ci véhiculent des représentations de genre équitables ou, au contraire, un "curriculum caché» (cf. Mosconi, 2010) qui minore ou dévalorise un de deux sexes. Et cela à travers une énonciation qui s'appuie sur des choix pragmatico-communicatifs et sur un environnement contextuel caractérisé par des représentations socioculturelles. Les notions de champs discursifs et de positionnements de l'analyse du discours s'avèrent donc pertinentes dans la configuration des équilibres et de l'identité énonciative, notamment à travers l'emploi du vocabulaire, du registre de langue, de tel genre de discours (Maingueneau, 2009 : 100).

20 Il est d'extrême importance de considérer également les images des manuels, ce que Bachelard appelle par « figures du monde » ou ce que Hjelmslev appelle « figures du plan de l'expression » (Greimas, $2001: 200$ ), ce qui permet de faire une lecture d'ordre figuratif des objets visuels cernant le caractère expressif et ce qu'ils produisent du point de vue iconique (cf. Sunderland, 2015).

21 Le micro-échantillon d'images concernant les unités thématiques sélectionnées, dont on ne reproduit ici que des exemples emblématiques, ne vise pas la représentativité statistique, mais plutôt une représentativité sur le plan sociologique qui s'appuie notamment sur le principe de saturation des données (Laperrière, 1994). 


\section{Représentations de genre dans les manuels de FLE}

\section{Famille}

La femme représentée est généralement située dans un contexte d'activités standardisées car consacrées à la famille alors que la famille constitue le prétexte pour apprendre des fonctions, des expressions liées à l'heure, aux différents moments de la journée au fil des activités quotidiennes; les activités évoquées montrent souvent une femme dans un rôle de ménagère (femme au foyer), située dans un espace isolé loin des activités de son partenaire :

Image 1 - «Observez ces vignettes et racontez la matinée type de Madame Durand » (Léonard, $2011: 57$ )

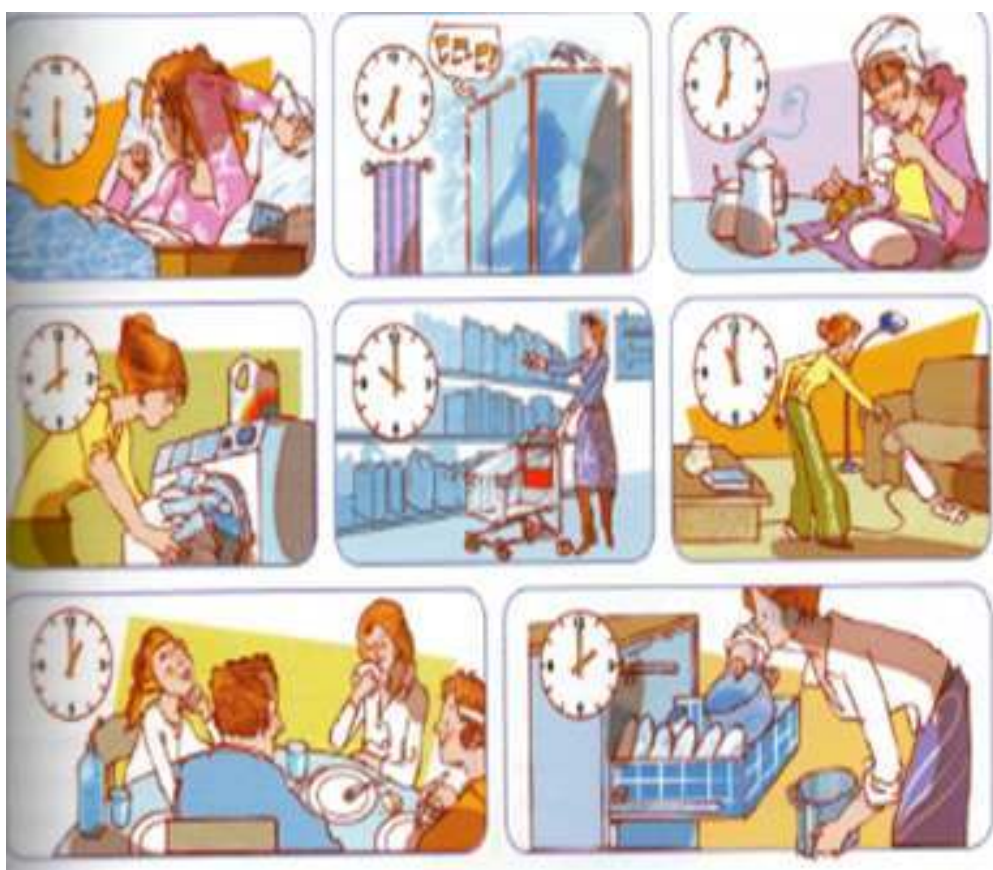

(c) Marco Bolla

Dans sa "matinée type ", Mme Durand s'occupe seule de toutes les tâches ménagères ; tout cela semble renforcer la catégorisation du genre de la femme dont le rôle est a priori bien circonscrit. Ce qui est intéressant à remarquer concerne la consigne de l'activité (Léonard, 2011 : 57) : «Observez les vignettes et racontez la matinée type de Madame Durand »; la présence du mot «type » renforce la généralisation des activités de la femme mariée: de plus, ce qui est intéressant au niveau sémiotique touche à une différence de positionnement de l'image de la femme selon les différents moments de travail : sa figure est essentiellement prise de profil, et peu expressive, lorsqu'elle est engagée dans son rôle de mère et de femme au foyer; une autre connotation semble s'établir dans l'utilisation des couleurs genrées stéréotypées : la femme est parfois en rose, tandis que l'homme est en bleu; vu de dos il domine cependant la scène en passant au premier plan. 


\section{Professions}

Dans le contexte d'apprentissage d'une langue destinée à des compétences professionnalisantes, le rôle des femmes semble être assez limité au niveau d'égalité des chances; l'ensemble des occurrences lexicales et iconographiques relevées indique que les femmes peuvent être journalistes, interprètes, diplomates ou directrices de recherche; les occurrences indiquent que les hommes peuvent occuper plusieurs charges importantes, se référant à des rôles institutionnels de grande responsabilité en tant qu'expert international, eurodéputé, commissaire européen, l'espace commun aux deux sexes se situe à un niveau intermédiaire, en tant qu'agents d'accueil, assistants ... (Riehl et al., 2006).

«Can the Subaltern speak?» interrogeait Spivak (1988); dans l'exemple suivant, la subalternité de la femme semble être explicite dans le discours des hommes entre eux :

À la cantine

Un monsieur seul cherche une place

Bruno Fantini : Excusez-moi, c'est libre?

Yves Sauvestre : Oui, oui, bien sûr, je vous en prie! Vous travaillez dans le bâtiment

$\mathrm{B}$ ?

Bruno Fantini : Oui, au service de la communication. Je viens d'arriver.

Yves Sauvestre : Ah bon. Vous êtes seul ou en famille?

Bruno Fantini: Seul, pour l'instant. Mais ma femme et mes enfants vont arriver le

30 septembre. Et vous?

Yves Sauvestre : Moi ? Je suis célibataire... Alors, vous aimez Bruxelles?

(Riehl et al., 2006: 36)

Le dialogue examiné se déroulant entre deux hommes tous les deux à Bruxelles pour des raisons professionnelles évoque la présence de la femme: «ma femme et mes enfants vont arriver ». La syntaxe de la phrase a fonction d'exemple pour l'apprentissage du futur proche, mais pour ce qui concerne notre analyse, l'implicite social s'oriente vers une représentation de la femme invisible dans un rôle d'accompagnatrice passive. Cette représentation participe à la construction de l'identité de genre et à son inscription dans le tissu professionnel et social : ici elle participe d'une légitimation de la mise en valeur de l'homme par la présence d'une famille qui le soutient et dont il détermine la mobilité.

\section{Achats}

27 Le thème des achats renforce lui-aussi une vision stéréotypée quant à la place occupée par la femme dans la société :

Leçon 9 « Ça me va comment?»

Camille - Salut, Sophie ! Je t'emmène dans une petite boutique qui se trouve rue des

Saints Pères. Ils ont des fringues hyper branchées! Il y a des tee-shirts rigolos, des ceintures de toutes les couleurs, des pulls originaux, des pantalons magnifiques...

Sophie - Ah bon!

(...)

Camille - Regarde ces chaussures ! Elles sont superbes, non?

Sophie - Lesquelles? Les rouges ou les noires?

Camille - Les rouges, celles qui coûtent 110 euros.

Sophie - Je les trouve quelconque et 110 euros, ce n'est pas donné.

Camille - On entre?

(...)

(Léonard, $2011:$ 107) 
Ce qui émerge de cet extrait concerne le stéréotype du "shopping" au féminin. Le dialogue qui vise fonctionnellement à introduire le vocabulaire lié aux vêtements, les pronoms démonstratifs, les pronoms interrogatifs, etc., met en scène l'attraction supposée toute féminine envers les vêtements, les accessoires. Dans l'exercice 3 du bilan annexe, "Écoutez le dialogue et complétez les phrases ", l'illustration (v. image 2) est particulièrement significative de ce stéréotype du fait qu'elle n'a pas justement pour fonction d'aider à la compréhension linguistique (les éléments chiffrés, pointures et prix, dans un dialogue entre une cliente et une vendeuse) mais évoque le désir d'acheter des bottes de type chic.

Image 2 - «Écoutez le dialogue et complétez les phrases » (Léonard, 2011 : 116)

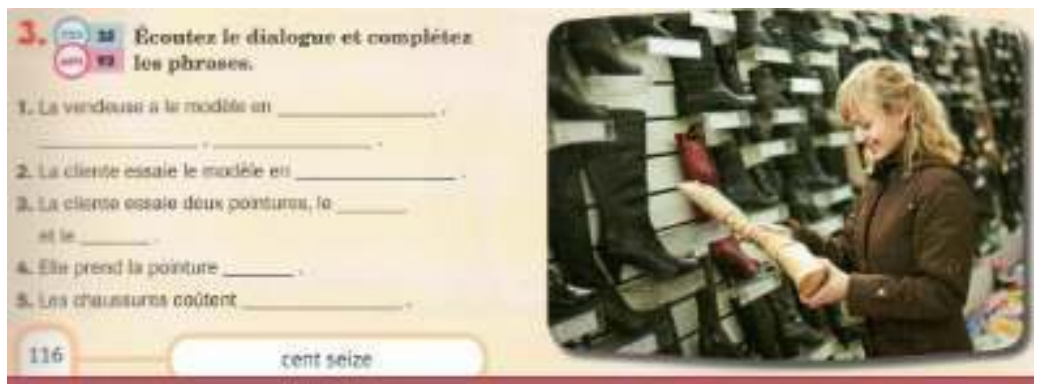

(c) Shutterstock

L'image semble renforcer le stéréotype de la frivolité et vanité supposées féminines en représentant une femme face à son objet de désir au beau milieu d'une profusion de modèles, dans une posture sémiotique figurative qui suggère chez la femme une identification complaisante avec la paire de bottes élégantes reprenant la couleur de ses cheveux.

\section{Représentations de genre dans les manuels d'italien langue étrangère et seconde}

\section{Famille}

30 L'image de la famille qui émerge de la plupart des manuels d'italien LE examinés est également assez stéréotypée surtout pour ce qui concerne la division des rôles. Dans les situations qui se rattachent à la vie domestique ou à la routine familiale, prédomine l'image d'une femme à tout faire qui s'occupe de la cuisine, du ménage, du repassage et de soigner les enfants, alors que les hommes sont souvent représentés comme étant moins préoccupés par la vie familiale. Les images sur les activités du dimanche de la famille Togni (v. image 3) en donnent un exemple : la femme passe un tablier et s'active dans le ménage alors que l'homme, ou le garçon, peut se consacrer à la lecture d'un journal ou s'occuper de tâches plus "masculines", comme tondre l'herbe. Si le temps libre de la femme est toujours inséré dans les interstices du calendrier familial, les hommes semblent donc avoir plus d'espace pour le soin de soi et de ses propres intérêts. 


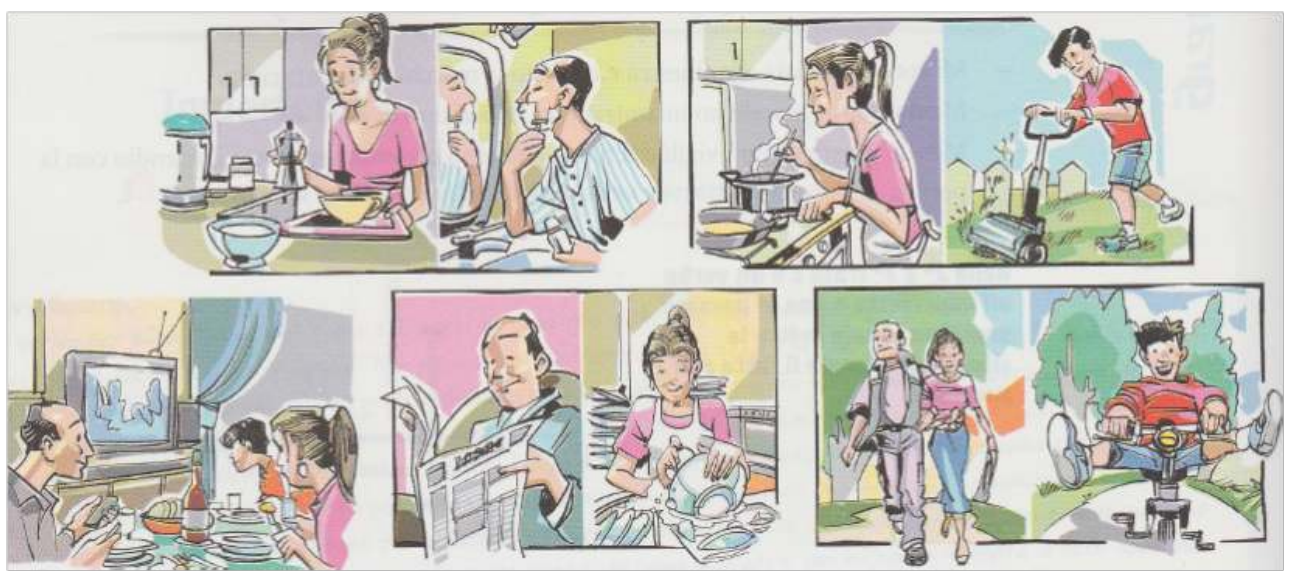

(c) Marco Francescato

Cette représentation stéréotypée des rôles familiaux et des actions routinières peut être renforcée par le choix du lexique, comme dans cet exercice proposé dans la même unité, où l'on remarque la présence d'une femme vouée à éplucher des pommes de terre ou installer les rideaux, tandis que l'homme est engagé à accrocher un tableau ou, au maximum, à égoutter les pâtes :

Mentre Gina (mettere)... le tende (cadere)... dalla scala e (farsi male)... a una gamba.

Mentre Lucio (scolare)... la pasta (scottarsi)... con l'acqua calda [...]

Mentre Giulia (pelare)... le patate (tagliarsi) ...

Mentre mio marito (appendere)... un quadro (martellarsi)... un dito ${ }^{13}$.

(Bozzone Costa et al., 2007 : 161)

Bien qu'une telle image de la famille traditionnelle soit encore très répandue dans le contexte socio-culturel italien, les références à un partage plus équitable des rôles en famille, à la présence de couples non mariés, ou de familles monoparentales restent très exceptionnelles.

\section{Professions}

Dans les manuels examinés, on peut observer une double iniquité dans la représentation des sexes en ce qui concerne les professions: sur le plan quantitatif, $62 \%$ des 96 occurrences lexicales et iconographiques sur les professions sont occupées par des hommes. En outre, les femmes exercent souvent des professions confinées à la sphère domestique ou des services: elles sont plus souvent ménagères, vendeuses, serveuses, secrétaires, coiffeuses, infirmières, couturières $(71 \%)$ ou, tout au plus, enseignantes $(13 \%$ ). L'homme occupe plus souvent des emplois libéraux professionnels (par ex. dentiste, médecin, journaliste, architecte [42\%]), des rôles de responsabilité (par ex. chirurgien, pédiatre, policier [17\%]), ou des emplois pénibles (par ex. maçon, mécanicien, ouvrier, etc. [10\%]). Même si dans le contexte privé, c'est le plus souvent la femme qui cuisine, lorsqu'il est question d'un rôle professionnel plus prestigieux tel que le chef d'un restaurant, c'est toujours la figure de l'homme qui l'emporte. L'asymétrie des rôles présentée dans le domaine personnel semble donc se répercuter et se renforcer dans le domaine professionnel se cristallisant dans une évidente dissymétrie sémantique qui voit les 
unités lexicales, liées aux rôles de plus grand prestige, se référer généralement aux personnages masculins.

Il semble donc prévaloir une représentation des femmes comme fragiles ou reléguées à des professions moins qualifiées et rémunérées. Par ailleurs, cette discrimination se poursuit $\mathrm{du}$ point de vue lexico-grammatical. Lorsqu'elles exercent des rôles professionnels de plus grand prestige, on utilise exclusivement le masculin générique ensembliste (il medico/le médecin, il chirurgo/le chirurgien), sans fournir aucune explication pour justifier à l'apprenant.e pourquoi on déroge aux règles de formation des noms italiens.

Cette représentation minorisante de la femme et de son rôle dans la société italienne semble se fossiliser à l'intérieur des manuels d'italien L2 à l'usage des femmes immigrées. Comme on peut observer dans l'image 4, représentant la situation « À l'hôpital », la seule concession faite au genre féminin est la profession de gynécologue, toutes les autres professions étant exercées par des hommes.

Image 4 - «Les professions à l'hôpital dans un manuel d'italien L2 »

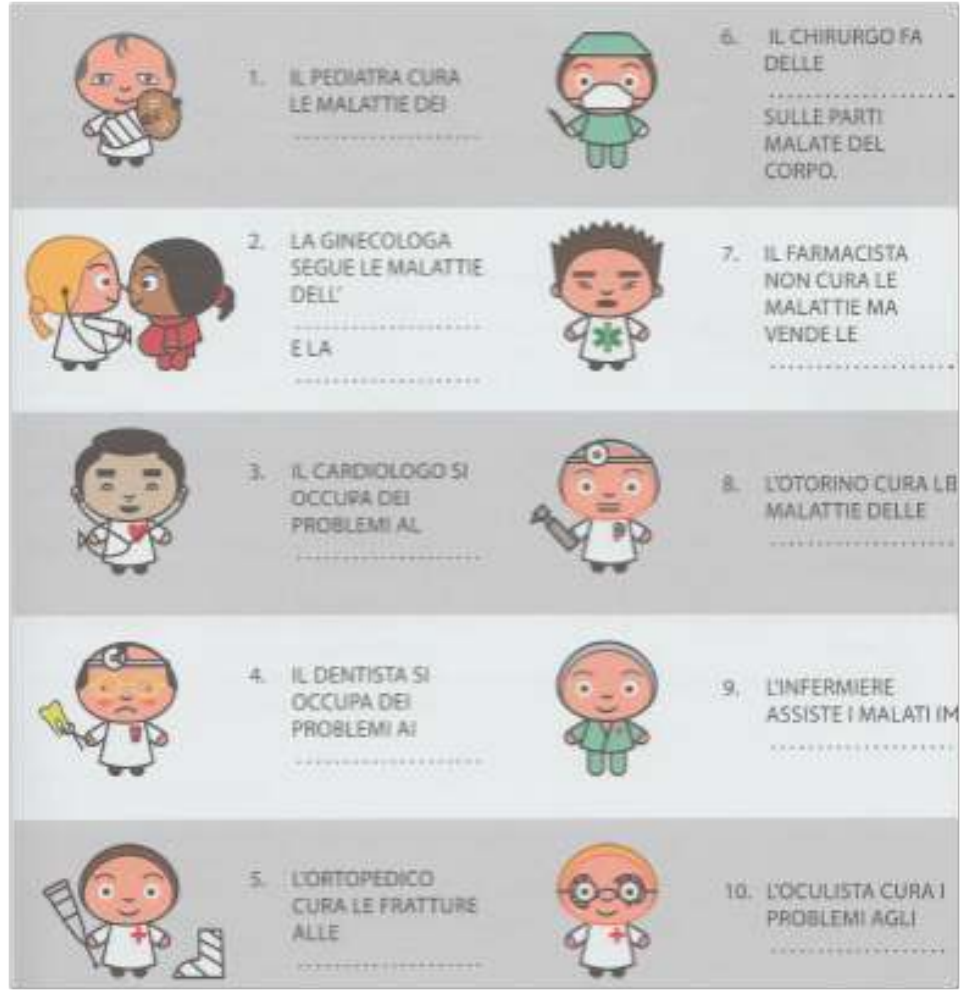

(Marelli \& Rodondi, 2013 : 95)

37 L'analyse sémiotique figurative des images permet de noter comment la représentation planaire et frontale des professions masculines contraste fortement avec celle de la gynécologue et de sa patiente qui se regardent réciproquement tout en souriant: la gynécologue est la seule spécialiste qui communique avec sa patiente, ici enceinte; cela renforce aussi la situation "type " où elle seule est habilitée à examiner l'immigrée présentée comme «typiquement » mère et musulmane. Alors qu'on croit aller dans le sens des besoins socioculturels de l'apprenante migrante, comme dans la situation donnée, on finit parfois par reproduire et durcir une vision stéréotypée, et au fond, très 
peu réaliste quant à la situation socioprofessionnelle actuelle en Italie, y compris pour ce qui est du taux de féminisation de la profession médicale.

\section{Achats}

Dans l'unité thématique "achats » on peut signaler une féminisation des thèmes aussi bien qu'une présence plus marquée de figures féminines : ce sont plus souvent les femmes qui occupent le rôle de vendeuses, qui achètent pour la préparation des aliments ainsi que des vêtements et des accessoires, comme dans cet extrait d'un dialogue qui voit comme protagonistes un jeune couple italien :

Dialogue « Un vestito per la festa di Marco »

Giada - Guarda quel vestito rosso, Massimo! Ti piace?

Massimo - Sì, ma quanto costa?

Giada - Solo 150 euro.

Massimo - 150 euro ? Ma costa una fortuna ! (.......)

Massimo - Giada ! 270 euro per un vestito e un paio di scarpe ! ?

Giada - Sì, ma sono perfetti insieme. Li compro. Qui accettano tutte le carte di credito.

Massimo - Va bene, ma almeno adesso possiamo andare? Non voglio passare tutto

il giorno a fare spese.

Giada - Uffa, sei sempre il solito ${ }^{14}$.

(Bettinelli et al., 2011 : 112)

Ce dialogue présente une représentation assez stéréotypée des rôles féminin et masculin et des relations homme/femme. Il est évident que Giada achète une robe et des chaussures à un prix assez élevé pour un événement ordinaire tel qu'une fête d'anniversaire ainsi que par rapport aux possibilités économiques d'un jeune couple. En plus, son copain lui reproche tout le temps d'avoir dépensé trop d'argent et de lui faire perdre son temps à faire du shopping.

Cette représentation assez frivole et dépensière de la femme italienne, d'ailleurs présente dans d'autres manuels du corpus, est renforcée dans la même unité didactique par l'emploi de données et graphiques statistiques qui, comme dans l'image 5, tente de légitimer l'importance de cette typologie d'achats pour les Italiens, et surtout les Italiennes, sans aucun détail sur la source des données employées comme gage de scientificité sociologique ${ }^{15}$.

Image 5 - "L'importance des achats des vêtements et des chaussures pour les Italiens » (Bettinelli et al., Buona idea !, Pearson Italia, 2011 : 117)

\section{L'importanza degli acquisti d'abbigliamento}

Per Lel è importante

tare acquisti

nel campo

dell'abbigllamento

e delle calzature?

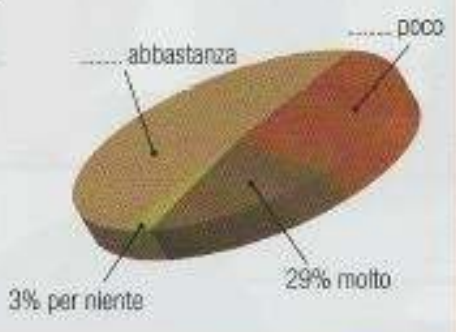




\section{Intérêts et loisirs}

41 Cette représentation frivole de la femme italienne est confirmée dans l'analyse de l'unité thématique «intérêts et loisirs » où les stéréotypes se référant aux filles semblent être encore plus marqués. Tout comme la femme au foyer qui n'a presque pas de temps libre, dans les manuels examinés on peut souvent retrouver l'image des filles qui occupent la plupart de leur temps à bavarder à propos de fêtes ou à organiser des fêtes, à faire du shopping ou, encore, des filles intéressées surtout par la télévision, la discothèque ou d'autres loisirs plus légers, comme cela est d'une façon emblématique résumé par les situations données dans les images 6 et 7 se référant à deux activités orales. L'écart qu'on établit entre les loisirs féminins (télévision, danse) et les loisirs de leurs homologues masculins (lecture, étude) semble être sans espace de convergence.

Image 6 - « temps libre et loisirs I » (Bozzone Costa et al., $2007: 47)$

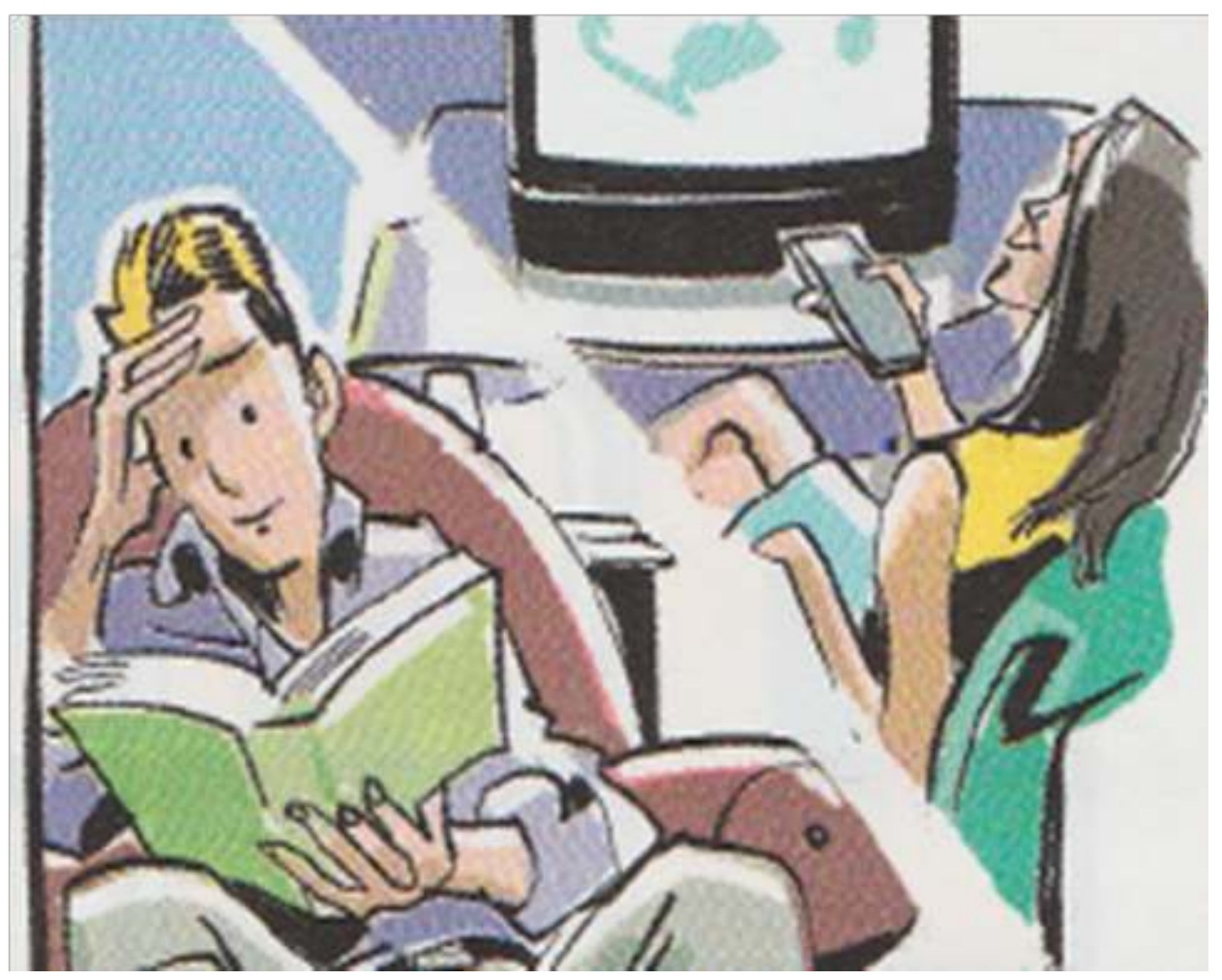

(c) Marco Francescato 


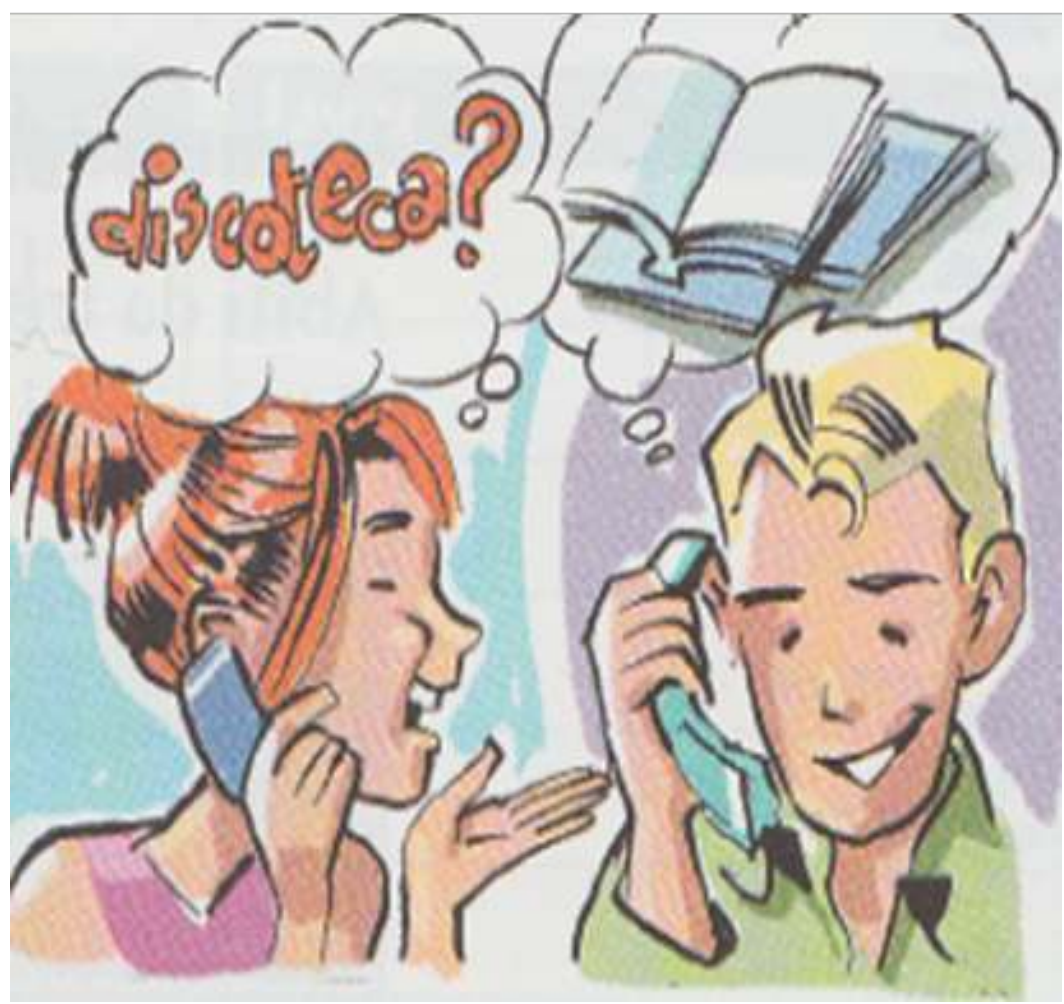

(c) Marco Francescato

Sur ces illustrations, la femme est représentée par derrière ou sur le côté, alors que l'homme est représenté de façon frontale, dans des attitudes réflexives, comme si on devait réaffirmer, sur le plan figuratif aussi, la plus grande importance de son rôle et de ses intérêts.

Bien que sur un mode mineur on peut noter la présence de représentations assez stéréotypées dans le domaine des activités sportives aussi : dans les exercices concernant le lexique du sport, par exemple, seulement les garçons jouent aux football, alors que ce sont surtout les filles qui jouent au volleyball ou font de la danse.

Les textes examinés disposent donc d'une vision tout aussi stéréotypée des centres d'intérêt des uns et des autres : d'une part, les filles et les femmes sont très fréquemment présentées comme soucieuses de leur aspect ou intéressées par des activités moins intellectuellement engagées; de l'autre part, les hommes et les garçons se révèlent amateurs de lecture ou d'activités de plein air ; à ce propos, l'ouvrage Papa lit, maman coud (Decroux-Masson, 1979), qui a fait son chemin, semble être malheureusement encore assez actuel.

\section{Repenser les stéréotypes de genre entre champ social et champ éducatif}

Un aperçu de la réalité éditoriale attachée au traitement du genre recèle des représentations insérées comme relevant de ce "qui va de soi ", sans que les neoenseignant.e.s soient toujours averti.e.s de la portée idéologique et réductive « du fait de 
leur méconnaissance de cette réalité » (Baurens, 2006: 244). Sans vouloir mettre en accusation le manuel de langue, on peut convenir que le discours converge vers des représentations genrées qui sous prétexte de faciliter l'accès au sens commun, sacralisent des stéréotypes.

À la lumière du corpus analysé, il semble donc fondamental de souligner l'importance d'un travail de déconstruction de ces représentations dans la formation initiale et continue des enseignant.e.s; ce processus assumé, les enseignant.e.s devraient, à leur tour, le réélaborer et proposer en classe, tout en s'interrogeant sur la place que le genre occupe aussi bien dans les représentations des élèves que dans leurs différentes languescultures de référence (cf. Baurens, 2009).

Si le choix du manuel n'est pas toujours du ressort de l'enseignant.e, il lui est toujours possible de contrer les représentations ordinaires en apportant des ressources complémentaires. En effet les dialogues font office dans le manuel de modèles discursifs, souvent à répéter pour mémoriser des structures linguistiques puis à réutiliser pour s'en approprier en recontextualisant les actes de langage dans des jeux de rôle. Un moment d'analyse de la situation langagière peut être utilisé pour déconstruire l'aspect genré des stéréotypes du manuel. La mise en situation par paires d'apprenant.e.s de même sexe de situations diverses à recréer comme jeux de rôles à représenter devant la classe, peut être l'objet d'une observation collective sur les différents actes de langage mis en jeu, sur l'aspect relationnel des échanges, sur les différences de sensibilité, de centres d'intérêt dans l'interprétation des actes sociaux selon des différences de genre, donnant à la classe de langue l'occasion de participer à l'interaction dans le respect des différences de façon réflexive et créative.

\section{Conclusion}

La spécificité de la didactologie/didactique des langues-cultures rend compte des potentialités de formation et d'autonomisation «d'acteurs de terrain directement concernés " au fur et à mesure qu'on met en place un travail constant de conceptualisation (Galisson, $2002: 268$ ). Du point de vue des perspectives de formation des enseignant.e.s, promouvoir une attitude sensibilisée à une approche intergenre à la didactique des langues-cultures apparaît comme fondamental ; les enseignant.e.s seraient eux/elles-mêmes incité.é.s à prendre conscience de leurs propres représentations du genre et en assumer les niveaux d'influence dans la relation didactique qui explicite toute action éducative.

Dans les termes de l'organisation de l'activité didactique et de ses modalités, les outils analytiques sélectionnés (v. \$ 2) pourraient faciliter un processus de re-contextualisation des stéréotypes de genre à partir d'un travail d'observation, de déconstruction et reconstruction des langages verbaux et non verbaux.

Enfin, à la lumière de la réflexion jusqu'ici proposée on souhaite que les documents des politiques européennes de formation - par exemple, le Portfolio Européen pour les enseignants en langues en formation initiale (Newby et al., 2008) - puissent inciter à un travail d'analyse et de révision de la dimension du genre afin de l'intégrer aux compétences constitutives de la formation ainsi que de la pratique pédagogico-didactique de l'enseignant.e des langues-cultures. 


\section{BIBLIOGRAPHIE}

\section{Études}

Baurens, M. (2006). « Différenciations sexuées en classes de langues-cultures : palimpseste du genre, nouvelle friche pour la didactologie des langues-cultures ». Études de Linguistique Appliquée, $n^{\circ}$ 142. pp. 239-255.

Baurens, M. (2009). «Vers une compétence transculturelle du genre dans la formation des enseignant-e-s ». Études de Linguistique Appliquée, n 152. pp. 429-442.

Biemmi, I. (2010). Educazione sessista. Stereotipi di genere nei libri delle elementari. Torino : Rosenberg \& Sellier.

Blanchet, A. \& Gotman, A. (1992). L'enquête et ses méthodes : l'entretien. Paris : Nathan.

Brugeilles, C. \& Cromer, S. (2008). Promouvoir l'égalité entre les sexes par les manuels scolaires. Un guide pour les acteurs et actrices de la chaîne du livre. Paris : Unesco.

Centre Hubertine-Auclert (2015). Manuels scolaires, genre et égalité. Actes du colloque du 2 juillet 2014, Paris. Consulté en juin 2017 : http://www.anef.org/wp-content/uploads/2014/06/ actes_04_manuelscolaire_22122014_web.pdf.

Commission Européenne (2010). Différences entre les genres en matière de réussite : étude sur les mesures prises et la situation actuelle en Europe. Strasbourg : EACEA.

Conseil de l'Europe (2001). Un cadre européen commun de référence pour les langues: apprendre, enseigner, évaluer. Paris : Didier.

Decke-Cornill, H. \& Volkmann, L. (2007). Gender studies and foreign language teaching. Tübingen : Narr.

Decroux-Masson, A. (1979). Papa lit, maman coud. Les manuels scolaires en bleu et rose. Paris : Denoël/ Gonthier.

Doise, W., Clemence, A. \& Lorenzi-Cioldi, F. (1995). Rappresentazioni sociali e analisi dei dati. Bologna : Il Mulino.

Galisson, R. (2002). « Préambule : est-il sage ? Est-il fou? ». Études de Linguistique Appliquée, n 127. pp. 262-271.

Galisson, R. (1994). « Formation à la recherche en didactologie des langues-cultures ». Études de Linguistique Appliquée, $\mathrm{n}^{\circ}$ 95. pp. 119-159.

Greimas, A.J. (2001). « Semiotica figurativa et semiotica plastica ». In Fabbri, P. \& Marrone, G. (dir.), Semiotica in nuce. Volume II. Teoria del discorso. Roma : Meltemi. pp. 196-210.

Laperrière, A. (1994). « Les critères de scientificité des méthodologies qualitatives ». In Conseil québécois de la recherche sociale (dir.), Les méthodes qualitatives en recherche sociale: problématiques et enjeux. Québec: Gouvernement du Québec. pp. 45-66.

Légal, J.-B. \& Delouvée, S. (2008). Stéréotypes, préjugés et discriminations. Paris : Dunod. 
Magno, C. \& Silova, I. (2007). « Teaching in transition. Examining school-based inequities in central/south-eastern Europe and the former Soviet Union ». International Journal of Educational Development, $\mathrm{n}^{\circ}$ 27. pp. 647-660.

Maingueneau, D. (2009). Les termes clés de l'analyse du discours. Paris : Seuil.

Matthey, M. (1997). Les langues et leurs images. Neuchâtel : IRDP Éditeur.

Mosconi, N. (2010). «Filles / garçons. Éducation à l'égalité ou transmission de stéréotypes sexistes? ». L'école et la ville, $\mathrm{n}^{\circ}$ 4. pp. 1-12.

Mustapha, A. \& Mills, S. (Ed.) (2015). Gender Representations in Learning Materials. International Perspectives. New York : Routledge.

Newby, D., Allan, R., Fenner, A.-B., Jones, B., Komorowska, H. \& Soghikyan, K. (2008). Portfolio européen pour les enseignants en langues en formation initiale. Un outil de réflexion pour la formation des enseignants en langues. Strasbourg, Conseil de l'Europe.

Nicola, F. (2016). « L'educazione alla parità di genere in Europa ». La ricerca, nº 10. pp. 39-42.

Pavlenko, A. (2004). « Gender and sexuality in foreign and second language education : Critical and feminist approaches ». In Norton, B. \& Toohey, K. (Ed.), Critical Pedagogy and Language Learning. Cambridge : Cambridge University Press. pp. 53-71.

Sapegno, M.S. (a cura di) (2010). Che genere di lingua ? Sessismo e potere discriminatorio delle parole. Roma : Carocci.

Sapegno, M.S. (a cura di) (2014). La differenza insegna. La didattica delle discipline in una prospettiva di genere. Roma : Carocci.

Schmenk, B. (2007). « Foreign Language Research and the Feminization of Language Learning ». In Decke-Cornill, H. \& Volkmann, L. (Ed.). pp. 121-134.

Serravalle Porzio E. (2000). Saperi e libertà : maschile e femminile nei libri, nella scuola e nella vita. Milano : Associazione Italiana Editori.

Sinigalia-Amadio, S. (2011). «Le genre dans les manuels scolaires français. Des représentations stéréotypées et discriminatoires ». Tréma, n 35-36. pp. 99-112.

Spivak, G. (1988). « Can the Subaltern speak?». In Nelson, C. \& Grossberg, L. (Ed.), Marxism and Interpretation of Culture. London : Macmilliam. pp. 24-28.

Sunderland, J. (2015). «Gender (Representation) in Foreign Language Textbooks : Avoiding Pitfalls and Moving On ». In Mustapha, A. \& Mills, S. (Ed.). pp. 19-34.

Zarate, G. (1997). « La notion de représentation et ses déclinaisons ». In Zarate, G. (dir.), Les représentations en didactique des langues et cultures. Notions en Question, vol. 2. Paris : Crédif-Didier. pp. 5-9.

\section{Manuels de langue cités}

Bettinelli, B., Della Putta, P. \& Visigalli, M. (2011). Buona idea! Corso di lingua e cultura italiana. Livello elementare. Milano-Torino : Pearson Italia.

Bozzone Costa, R., Ghezzi, C. \& Piantoni, M. (2007). Contatto 1. Corso d'italiano per stranieri. Torino : Loescher.

Léonard, M. (2011). Français.ado. Méthode de français. Livre de l'élève-Apprentissage individuel. Torino : Loescher. 
Marelli, M. \& Rodondi, F. (2013). Parole in movimento. Percorso di alfabetizzazione e educazione alla cittadinanza. Bergamo : Sestante.

Riehl, L., Soignet, M. \& Amiot, M.-H. (2006). Objectif diplomatie. Le français des relations européennes et internationales A1/A2. Paris : Hachette.

\section{NOTES}

1. La contribution a été rédigée en étroite collaboration par les auteures; toutefois Edith Cognigni s'est chargée plus particulièrement de l'introduction et des $\S 1,4,5$, Silvia Vecchi des $\S$ 2,3 et de la conclusion.

2. «Le terme équité est utilisé pour désigner une situation où les élèves reçoivent la même qualité d'enseignement et profitent du même type d'équipements scolaires, manuels et ressources d'enseignement. [...] Par contre, l'équité de genre se fonde sur le principe de la justice sociale et se réfère à la garantie de résultats éducatifs équitables, indépendamment des différences de sexe. » [trad. par nos soins].

3. Dorénavant LS/LE.

4. Quant au concept général de stéréotype, v. Légal \& Delouvée, 2008. Pour le concept de stéréotype de genre v. Mustapha \& Mills, 2015.

5. L'acronyme italien POLITE en français veut dire Égalité des chances dans les manuels scolaires.

6. En particulier, on y recommande: a) d'éviter le sexisme et les stéréotypes sexuels ; b) d'assurer une représentation équilibrée des différences ; c) de promouvoir la formation d'une culture de la différence entre les sexes; d) de repenser la langue ; e) de mettre à jour et d'adapter le choix des illustrations.

7. Pour d'autres actions réalisées dans le champ éducatif italien, v. par ex. Sapegno 2010 et 2014.

8. V. par exemple Brugeilles \& Cromer, 2008; Centre Hubertine-Auclert, 2015 ; Sinigalia-Amadio, 2011.

9. La notion de « représentation sociale » ici utilisée s'inspire des études de Willem Doise (1995) à côté de celles de Geneviève Zarate (1997) et de Marinette Matthey (1997) que nous retenons pour la spécificité du champ de la didactique des langues.

10. La période de publication des textes a été définie afin d'évaluer le niveau de réception des recommandations du Code d'Autoréglementation et des Vademecum (supra, § 1), notamment par rapport aux manuels publiés en Italie.

11. Pour certaines unités thématiques, on a prévu aussi une approche quantitative (par ex. comptage d'occurrences lexicales ou iconographiques), afin de souligner de manière plus évidente certaines formes d'iniquité de genre émergent du corpus (v. § 4.2).

12. Pour les limites objectives d'espace, on mettra l'accent sur les niveaux de l'analyse thématique et figurative sémiotique en donnant quelques exemples significatifs au niveau de l'énonciation du discours.

13. - Pendant que Gina (installer) ... les rideaux (tomber) ... de l'escalier et (se blesser) ... à une jambe.

- Pendant que Lucio (égoutter les pâtes) ... (se bruler) ... avec l'eau chaude [...]

- Pendant que Julia (éplucher) ... les pommes de terre (se couper) ...

- Pendant que mon mari (accrocher) ... un tableau (se marteler) ... un doigt. [trad. par nos soins]

14. Dialogue « Une robe pour le parti de Marco »

Giada - Regarde cette robe rouge, Massimo! Tu l'aimes?

Massimo - Oui, mais ça coûte combien?

Giada - Seulement 150 euros.

Massimo - $150 €$ ? Mais ça coûte une fortune! (....) 
Massimo - Giada! $€ 270$ pour une robe et une paire de chaussures?

Giada - Oui, mais ils sont parfaits ensemble. Je les achète (...)

Massimo - D'accord, mais au moins maintenant nous pouvons y aller? Je ne veux pas passer toute la journée à faire du shopping.

Giada - Oh là là, c'est toujours pareil avec toi. [trad. par nos soins].

15. L'importance des achats des vêtements (titre). Pour vous, est-il important de faire des achats dans le champ des vêtements et des chaussures ? (sous-titre) assez; peu ; beaucoup: $29 \%$; pas du tout : $3 \%$ (graphique). [trad. par nos soins]

\section{RÉSUMÉS}

Axée sur la relation entre genre et didactique des langues-cultures, notre contribution a pour but d'approfondir la notion de "stéréotype de genre " à l'aide d'une analyse portant sur des matériaux didactiques sélectionnés pour l'enseignement de l'italien et du français langues secondes ou étrangères. À la lumière de cette analyse, on souhaite ouvrir quelques perspectives pour la formation des enseignant.e.s de langues afin de les placer en condition de reconnaître et de déconstruire les représentations de genre et les langages sexistes ainsi que de se faire agents d'une éducation linguistique attentive à l'équité de genre.

Focused on the relationship between gender and language and culture teaching, this paper aims at deepening the concept of "gender stereotype " by analyzing selected materials for the teaching of Italian and French as second or foreign languages. In light of this analysis, we are proposing some perspectives for the training of language teachers in order to put them in the condition to recognize and deconstruct gender representations and sexist language, and thus to become agents of a gender-sensitive language education.

\section{INDEX}

Thèmes : Varia

Mots-clés : enseignement des langues-cultures, représentation du genre, équité de genre, formation des enseignant.e.s

Keywords : language and culture teaching, gender representation, gender equity, teacher training

\section{AUTEURS}

\section{EDITH COGNIGNI}

Edith Cognigni est docteure de recherche en « Politique, Éducation, Formation LinguisticoCulturelles » et enseignante-chercheure en Didactique des langues au Département d'Études Humanistes de l'Université de Macerata (Italie). Ses domaines de recherche portent sur l'apprentissage/enseignement de l'italien langue seconde en contexte migratoire, le genre en didactique des langues-cultures, l'intercompréhension et l'interaction plurilingue à distance. 
http://docenti.unimc.it/edith.cognigni

edith.cognigni[at]unimc.it

Université de Macerata, Italie

\section{SILVIA VECCHI}

Silvia Vecchi est docteure de recherche en « Politique, Éducation, Formation LinguisticoCulturelles ». Ses domaines de recherche portent sur la didactique des langues-cultures, la formation des enseignants (FLE) et la pluri-/interdisciplinarité en didactique des languescultures.

silvia.vecchi[at]unimc.it

Université de Macerata, Italie 